\title{
Utilidad de la Plataforma "Pruéb@te UNAM Licenciatura” para Ingresar a la Educación Superior
}

\section{Usefulness of the "Pruéb@te UNAM" Platform for Admission to Higher Education}

Melchor Sánchez-Mendiola *, Sandra Paola Rodríguez Castillo, Nancy Fabiola Pérez Herrera, Manuel García-Minjares, Adrián Martínez-González

Universidad Nacional Autónoma de México, México

\section{DESCRIPTORES:}

Examen de ingreso

Aprendizaje en línea

Evaluación sumativa

Examen práctico

Selección de estudiantes

\section{RESUMEN:}

El proceso de admisión a la educación superior en varias universidades incluye exámenes estandarizados de alto impacto, que se asocian a preparación especial para ayudar a los aspirantes a dominar estrategias para resolución de pruebas, así como revisar y practicar los conocimientos evaluados. El objetivo de este estudio fue comparar el rendimiento en el examen de ingreso a las licenciaturas de la Universidad Nacional Autónoma de México (UNAM) de los usuarios de la plataforma digital "Pruéb@te UNAM Licenciatura” versus los no usuarios, así como establecer la relación entre uso de la plataforma y ser seleccionado. Se realizó un estudio cuantitativo observacional, comparativo y transversal. La población del estudio correspondió a 178.364 aspirantes a los concursos de selección en 2018 de la UNAM, de estos 8.063 fueron usuarios de la plataforma. Los usuarios tuvieron mayor puntuación en el examen de ingreso que los no usuarios $(\mathrm{p}<0,001 ; \mathrm{d}=0,76)$. Practicar más de 10 horas $(\mathrm{p}<$ $0,001 ; d=0,89)$ y completar hasta cinco exámenes de prueba $(\mathrm{p}<0,001 ; \mathrm{d}=0,88)$, se asociaron con resultados superiores. Utilizar una plataforma de preparación se asocia con una mayor puntuación en el examen de ingreso a la universidad. El aprendizaje potenciado por exámenes puede ser útil para entender y mejorar el desempeño en exámenes sumativos de alto impacto.

\section{KEYWORDS:}

Entrance examinations

Computer assisted learning

Practical examinations Summative evaluation Student selection

\section{ABSTRACT:}

The admission process to higher education in several universities includes standardized high-stakes examinations, that frequently are associated with special preparation courses designed to help applicants to master test-taking strategies, as well as to review and practice the knowledge assessed in the test. The goal of this study was to compare the performance in the college admission exam to the National Autonomous University of Mexico (UNAM) in users of the digital platform "Pruéb@te UNAM Licenciatura" versus non-users, and to establish the correlation between being selected and platform use. A quantitative observational, comparative and cross-sectional design was used. The study population was integrated by 178.364 applicants for a college career at UNAM, of these 8.063 used the platform. Users had higher scores in the admission exam than non-users $(\mathrm{p}<0,001 ; \mathrm{d}=0,76)$. Practicing more than 10 hours $(p<0,001 ; d=0,89)$ and completing up to five mock exams $(p<0,001 ; d$ $=0,88$ ) were associated with higher results. The use of a platform for training and rehearsal in simulated tests is associated with a higher score on the university admission exam. Test-enhanced learning can be useful to understand and improve applicants' performance in high-stakes summative examinations.

CÓMO CITAR:

Sánchez-Mendiola, M., Rodríguez Castillo, S. P., Pérez Herrera, N. F., García-Minjares, M. y Martínez-González, A. (2021). Utilidad de la plataforma "Pruéb@te UNAM licenciatura" para ingresar a la educación superior. Revista Iberoamericana de Evaluación Educativa, 14(2), 21-36. https://doi.org/10.15366/riee2021.14.2.002

*Contacto: melchorsm@unam.mx

ISSN: 1989-0397

https://revistas.uam.es/riee 


\section{Introducción}

En México ha aumentado la cobertura en educación desde el nivel básico hasta el nivel superior, sin embargo, solo el 56,3\% de los mexicanos concluyen el nivel de educación media superior, de acuerdo con datos proporcionados por la Organización para la Cooperación y el Desarrollo Económicos (OECD, 2018), quienes en su mayoría buscan ingresar a la educación superior. La Secretaría de Educación Pública de México (SEP, 2019) señala que para el ciclo escolar 2018/19 la matrícula total del nivel Licenciatura fue de 3.610.744 estudiantes inscritos en la modalidad escolarizada en instituciones mayoritariamente públicas.

El acceso a Educación Superior en México, principalmente en instituciones públicas, involucra la aplicación de exámenes estandarizados para identificar el nivel de dominio en áreas de conocimiento específicas, los cuales son elaborados por las propias instituciones educativas, como es el caso de la Universidad Nacional Autónoma de México (UNAM), la Universidad Autónoma Metropolitana (UAM) o el Instituto Politécnico Nacional (IPN), o por centros de evaluación como el Examen Nacional de Ingreso (EXANI II) del Centro Nacional de Evaluación para la Educación Superior. En Estados Unidos de América se cuenta con pruebas que se toman en cuenta en la selección de candidatos, una de ellas es el Scholastic Aptitude Test (SAT) que explora matemáticas, lectura crítica y escritura. Una más es el American College Test (ACT), la cual consta de cuatro áreas: inglés, matemáticas, lectura, ciencias y escritura (Briggs, 2009).

Dichos procesos de selección, por su impacto en la vida de los estudiantes, con frecuencia propician la aparición de diversos programas o cursos de preparación y entrenamiento especiales para mejorar su desempeño en los exámenes de admisión. De esta manera, en el presente trabajo se analizó si la preparación previa a través de la plataforma digital "Pruéb@te UNAM Licenciatura" se asocia con diferencias en rendimiento de los sustentantes en el examen de ingreso a la UNAM.

\section{Revisión de la literatura}

En los Estados Unidos existe una cantidad abundante de literatura publicada sobre los cursos de preparación para ingresar a la universidad, que documentan que tienen efectos positivos (Montgomery y Lilly, 2012), situación que contrasta con los países de economías emergentes como México, en los que hay escasa investigación sobre el tema. Prácticamente en todos los países que tienen exámenes sumativos de alto impacto como mecanismo de selección para ingresar a la universidad, para ingresar a posgrados como especialidades médicas, o para certificarse en el ejercicio de la profesión o una especialidad, hay una gran oferta de cursos presenciales, en línea o mixtos que, con tarifas relativamente elevadas, son adquiridos por muchos de los aspirantes, con el fin de mejorar sus puntuaciones e incrementar la probabilidad de ser seleccionado (McGaghie et al., 2004). Este tipo de cursos y herramientas para entrenarse para el examen, forman parte de la realidad cotidiana de los estudiantes en nuestro país y, aunque las empresas que venden el acceso a este tipo de recursos garantizan elevar sus puntuaciones, no se encontraron publicaciones formales en los que se analice con rigor metodológico su efectividad, solo se tiene información de tipo anecdótico de quienes los han tomado (Sanchez-Mendiola y Delgado-Maldonado, 2017).

La preparación o entrenamiento especial pretende fortalecer a los aspirantes en el reconocimiento del tipo y características de la prueba que se les aplicará, familiarizarse con los contenidos e identificar pistas en las preguntas de los exámenes para dar con la respuesta correcta (Powers, 1999; Winke y Lim, 2017). El supuesto es que los estudiantes que reciben preparación tendrán un mejor rendimiento en el examen de admisión comparado con los que no la recibieron (Briggs, 2009; Carrasco, 2011), aunque el objetivo principal de la preparación no sea la admisión sino favorecer el rendimiento en los futuros cursos universitarios (Rosefsky et al., 2016).

Estudios realizados para analizar los efectos de la preparación o entrenamiento para enfrentar evaluaciones sumativas de alto impacto, han utilizado diferentes metodologías para identificar su efectividad. En algunos casos examinan los cambios en el puntaje de los alumnos que cursaron un programa de preparación, en otros, se realiza la comparación de quienes lo tomaron con respecto a los que no recibieron entrenamiento, finalmente, algunos estudios utilizan el metaanálisis como técnica analítica para examinar los efectos a través de un gran número de estudios individuales, que pueden no ser comparables debido a diferencias en diseño, muestras y condiciones (Powers, 1999; Sanchez, 2019). 
$\mathrm{Al}$ analizar los resultados del entrenamiento o programas de preparación y su relación con diferentes variables, se identificó que la efectividad es independiente del género, el nivel socioeconómico o el tipo de escuela a la que asisten los alumnos (pública o privada). En este sentido, Appelrouth y otros (2017) encontraron que la preparación para la prueba SAT entre hombres y mujeres parece ser neutral, aunque las mujeres completaron significativamente más tareas y pruebas de simulación que los hombres, el aumento en la puntuación fue casi idéntico en ambos grupos. Cuando compararon alumnos por nivel socioeconómico alto y bajo, encontraron que los del grupo alto lograron mejor puntaje en cada sección del examen. En cuanto al tipo de escuela, los estudiantes de escuelas públicas alcanzaron puntajes más bajos y aumentos menores que sus contrapartes de escuelas privadas. Sin embargo, en el modelo lineal general, cuando se consideraron otros factores, la distinción entre escuela pública y privada resultó insignificante.

Adicionalmente, se han explorado factores de la preparación o entrenamiento que contribuyen a mejorar el rendimiento en los exámenes. Así, el tiempo de dedicación en los cursos de preparación correlaciona positivamente con el aumento en la puntuación y disminuye la ansiedad académica. Las pruebas prácticas o de simulación optimizan la retención, el aprendizaje en general y los resultados finales, además, la realimentación en estas pruebas juega un papel importante porque ayuda a los estudiantes a fortalecer su comprensión y minimizar el exceso de confianza (Appelrouth et al., 2017).

También se ha encontrado que la presentación repetida de la prueba favorece la familiaridad con los contenidos, por tanto, se obtienen ganancias sustantivas de conocimiento y en cuanto a las formas de preparación, aquellos estudiantes que contaron con tutoría individual o tomaron clases en cursos privados obtuvieron puntuaciones mayores (Appelrouth y Zabrucky, 2017).

En este orden de ideas, aunque los exámenes generalmente son vistos predominantemente como dispositivos de evaluación, también promueven el aprendizaje y aumentan directamente la retención duradera de información, esto es, los estudiantes que son evaluados acerca de un material lo recuerdan mucho mejor que cuando no se les evalúa o cuando usan otros métodos como lectura repetida del material, subrayar, entre otros. Este fenómeno es conocido como "aprendizaje potenciado por la prueba" (test-enhanced learning, en inglés), el cual se estimula con exámenes que se realizan frecuentemente con preguntas que requieren la producción de respuestas más que solo reconocimiento, junto con realimentación que ayude a los estudiantes a corregir errores y confirmar las respuestas correctas, y favorecer así el aprendizaje, la retención y transferencia de conocimientos (Green et al., 2018; Larsen et al., 2008; Leeming, 2002). Estudios de psicología cognitiva han documentado que examinar repetidamente a los estudiantes produce una retención superior de la información, comparado con el estudio repetido al que estamos tan acostumbrados, sobre todo cuando las pruebas son espaciadas en el tiempo (Brame y Biel, 2015; Larsen et al., 2008; McConnell et al., 2015).

Tomando en cuenta que la práctica con exámenes frecuentes puede tener un impacto sustancial en el aprendizaje, es deseable desarrollar recursos gratuitos o de bajo costo que faciliten a los estudiantes el estudio del material al que se enfrentarán en los exámenes de alto impacto. En este sentido, la UNAM creó la plataforma "Pruéb@te UNAM Licenciatura" como una herramienta que permite al usuario estudiar a su propio ritmo y con ello contribuir a la preparación para el examen de ingreso a las licenciaturas.

\subsection{Plataforma "Pruéb@te UNAM Licenciatura"}

La UNAM cuenta con dos recursos de preparación para presentar el examen de admisión a las licenciaturas: un documento impreso que es una Guía de estudio para el ingreso a la Licenciatura y una herramienta en línea denominada "Pruéb@te UNAM Licenciatura", disponible en la página http://www.pruebate.unam.mx.

Pruéb@te es una aplicación desarrollada por la Dirección de Evaluación Educativa (DEE), área de la Coordinación de Universidad Abierta, Innovación Educativa y Educación a Distancia (CUAIEED) de la UNAM, en la que se integra tanto la evaluación para el aprendizaje como los recursos tecnológicos, con la finalidad de que los usuarios estudien de manera autogestiva desde cualquier dispositivo con acceso a Internet.

$\mathrm{Al}$ ingresar a la plataforma, los usuarios completan un formulario de registro que los dirige a los contenidos que pueden estudiar según el área de conocimiento y licenciatura que desean cursar. Pruéb@te cuenta con tres módulos, dos de ellos permiten la autoevaluación y el refuerzo de conocimientos, a través de la Práctica de exámenes, donde los usuarios resuelven ejercicios similares al examen de selección de la licenciatura y el módulo de Práctica de materia, en el que trabajan con las asignaturas y temas donde presentan un bajo desempeño. Finalmente, en el apartado de Recursos y bibliografía, los estudiantes tienen acceso a literatura, ligas y 
otros sitios relacionados con los temas de los programas académicos que se encuentran en la red y que aportan mayor información aprobada por el Consejo Académico del Bachillerato (CAB) de la UNAM (Buzo et al., 2018).

\subsection{Pregunta de investigación y objetivos del estudio}

La pregunta de investigación que se planteó fue la siguiente: ¿cuál es el rendimiento en el examen de selección de los aspirantes que utilizaron Pruéb@te UNAM Licenciatura en comparación con los que no la usaron?

Los objetivos del estudio fueron: Comparar el rendimiento en el examen de ingreso a la UNAM de los usuarios de Pruéb@te UNAM Licenciatura versus los no usuarios, así como establecer la relación entre el uso de la plataforma y ser o no seleccionado, con sexo, edad, tipo de sostenimiento, bachillerato, área de conocimiento, nivel de demanda de la carrera, número de exámenes completados en Pruéb@te y el tiempo de uso de la plataforma (horas de práctica).

\section{Método}

\section{Diseño}

Se realizó un estudio cuantitativo observacional, comparativo y transversal (Manterola y Otzen, 2014), en el que se comparó el rendimiento de los usuarios de Pruéb@te UNAM versus los que no lo utilizaron, de la población de aspirantes que presentaron examen de admisión en el proceso de ingreso a las licenciaturas de la UNAM en el concurso de 2018.

\section{Población}

La población del estudio correspondió a 178.364 aspirantes a los concursos de selección en 2018 de la UNAM, de los cuales 8.063 fueron usuarios que por decisión propia utilizaron la plataforma digital Pruéb@teUNAM Licenciatura.

\section{Variables}

Las variables consideradas en el estudio fueron: sexo, edad, tipo de sostenimiento, bachillerato, área de conocimiento, nivel de demanda de la carrera, número de exámenes completados en Pruéb@te, el tiempo de uso de la plataforma (horas de práctica) y porcentaje de aciertos en el examen de selección para el ingreso a las licenciaturas 2018.

\section{Procedimiento y recolección de información}

Para el concurso de selección 2018 de las licenciaturas del Sistema Escolarizado de la UNAM se publicaron dos convocatorias cuyos respectivos exámenes se realizaron en febrero y junio de ese año. Para las carreras del Sistema de Universidad Abierta y Educación a Distancia (SUAyED) de la UNAM, se agrega una convocatoria con un examen en noviembre. Pruéb@te UNAM Licenciatura estuvo disponible todo el año 2018 para cualquier aspirante que quisiera usarlo, en ese lapso, los usuarios tuvieron acceso a los tres módulos de estudio.

El proceso de recolección de información se realizó en enero de 2019, una vez que concluyeron las actividades de las convocatorias y se tuvieron los resultados de sus respectivos exámenes. Por un lado, se recuperaron los registros de aspirantes a la UNAM de las convocatorias de 2018, de los cuales se obtuvieron las calificaciones globales obtenidas por los usuarios de Pruéb@te UNAM en el último examen aplicado. Por otro, se consultaron las bases de datos de los módulos de Práctica de exámenes y Práctica de materia del periodo comprendido de enero a diciembre de 2018, las cuales están a cargo de la Dirección de Evaluación Educativa (DEE), a fin de identificar el número de exámenes de práctica y el número de materias en las que estudiaron los usuarios de la Web App. Se consideró el nivel de demanda para las distintas licenciaturas a partir de tres aspectos: carrera, plantel y sistema, los cuales se utilizaron para la clasificación en cinco categorías (Muy baja, Baja, Media, Alta y Muy alta demanda). 


\section{Análisis estadístico}

Se calcularon medidas de tendencia central y de dispersión para el porcentaje de aciertos en el examen de ingreso en cada uno de los grupos de estudio (usuarios vs no usuarios) en cada una de las variables de interés; para determinar diferencias estadísticamente significativas se empleó la prueba t de Student, análisis de varianza (ANOVA) y el modelo de regresión lineal múltiple. Se realizó el cálculo de la d de Cohen para determinar el tamaño del efecto de las diferencias.

\section{Resultados}

\subsection{Rendimiento de los usuarios y no usuarios en el examen de ingreso 2018 de la UNAM}

En el Cuadro1 se observa que la media del porcentaje de aciertos en el examen de ingreso obtenida por los usuarios de Pruéb@te fue de 59,6, mientras que en los no usuarios fue de 48,3 ( $p<0,001)$. El tamaño del efecto de la diferencia (d) fue de 0,76 .

Cuadro 1

Porcentaje de aciertos obtenido por los aspirantes en el examen de ingreso en los concursos de selección 2018 de la UNAM, usuarios vs no usuarios de Pruéb@te

\begin{tabular}{lccccc}
\hline & $\mathrm{N}$ & $\%$ & Media & D.E. & $\mathrm{d}$ \\
\hline Usuarios & 8.063 & 4,5 & $59,6^{*}$ & 16,2 & \\
No usuarios & 170.301 & 95,5 & 48,3 & 14,6 & \\
\hline Total & 178.364 & 100,0 & 48,8 & 14,9 & 0,76 \\
\hline
\end{tabular}

Notas. ${ }^{*} \mathrm{t}=63,1 ; \mathrm{p}<0,001$. Se excluyeron del análisis 26.236 casos $(12,8 \%)$ sin datos.

La Figura 1 muestra los polígonos del porcentaje promedio de aciertos obtenido por los usuarios y no usuarios de Pruéb@te. En la distribución se observa un desplazamiento a la derecha del polígono que corresponde a los usuarios, en este sentido, a partir del $51 \%$ de aciertos son los usuarios de la plataforma los que logran el porcentaje promedio más alto.

\section{Figura 1}

Polígono de porcentaje de aciertos obtenidos por los aspirantes usuarios vs no usuarios de Pruéb@te en el examen de ingreso 2018

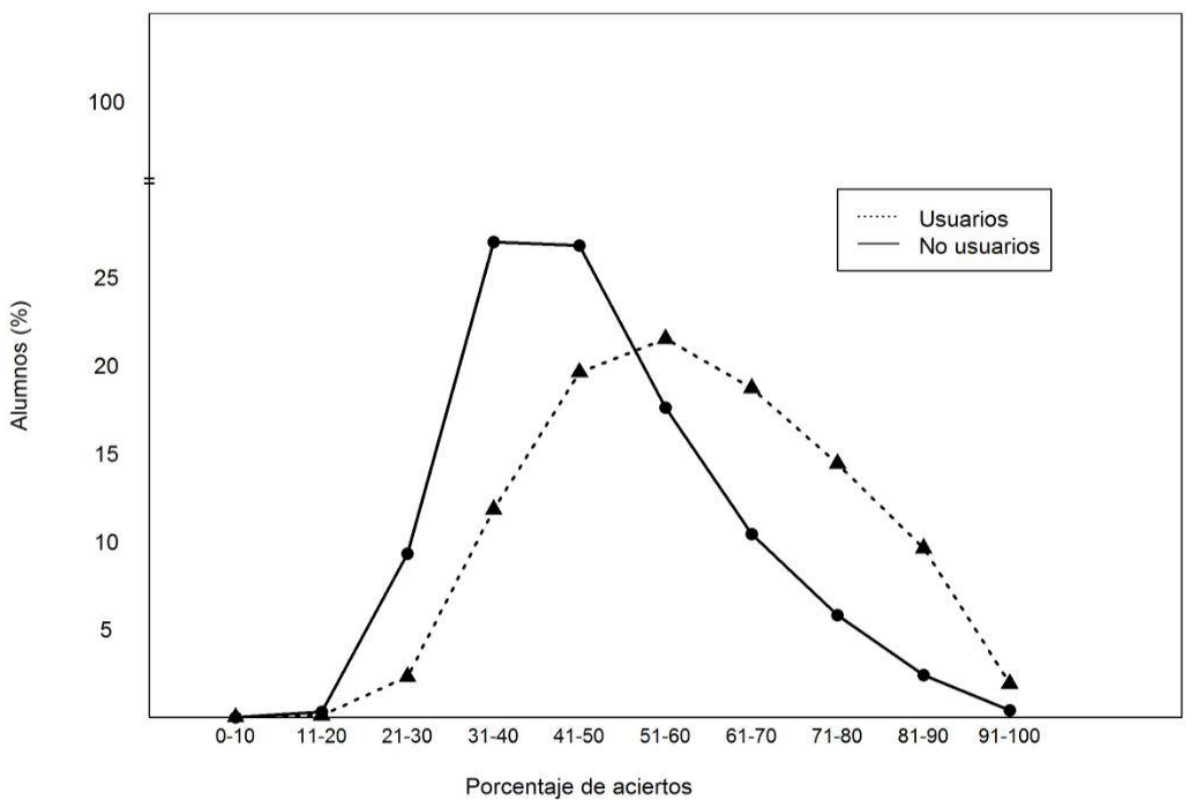


Comparación del rendimiento en el examen de ingreso 2018 de la UNAM de los usuarios y no usuarios, por sexo

De los 8.063 usuarios de Pruéb@te que aplicaron a alguna licenciatura de la UNAM, el 55,2\% fueron mujeres y $44,8 \%$ hombres. La distribución por sexo para los no usuarios fue de $55,3 \%$ y $44,7 \%$, respectivamente. La diferencia en desempeño en la prueba entre usuarios y no usuarios por sexo resultó significativa, los usuarios hombres obtuvieron un porcentaje medio de aciertos mayor $(62,4 \%)$ que sus homólogos no usuarios $(50,2 \%)$ con un tamaño del efecto de $\mathrm{d}=0,79$, mientras que las mujeres usuarias obtuvieron $57,3 \%$ de aciertos comparadas con las no usuarias, que fue de $46,7 \%$ (Cuadro 2).

Comparación del rendimiento en el examen de ingreso 2018 de la UNAM de los usuarios y no usuarios, por edad

Como se muestra en el Cuadro 3, las diferencias entre los usuarios y no usuarios según la edad resultaron significativas. Se observaron efectos grandes en el rango de menos de 18 y hasta 19 años $(\mathrm{d} \geq 0,90)$. Adicionalmente, los usuarios de 19 años obtuvieron el porcentaje medio de aciertos más alto (64\%), mientras que en los no usuarios se encontró en el nivel de 20 años $(50,4 \%)$.

Cuadro 2

Porcentaje de aciertos obtenido por los aspirantes usuarios vs no usuarios de Pruéb@te en el examen de ingreso en los concursos de elección 2018 de la UNAM, por sexo

\begin{tabular}{|c|c|c|c|c|c|c|c|c|c|c|c|c|c|}
\hline & \multicolumn{4}{|c|}{ Usuarios } & \multicolumn{4}{|c|}{ No usuarios } & \multicolumn{4}{|c|}{ Total } & \multirow{2}{*}{ d } \\
\hline & $\mathrm{N}$ & $\%$ & M & $\mathrm{DE}$ & $\mathrm{N}$ & $\%$ & M & $\mathrm{DE}$ & $\mathrm{N}$ & $\%$ & M & $\mathrm{DE}$ & \\
\hline Mujeres & 4.450 & 55,2 & $57,3^{1 *}$ & 15,7 & 94.257 & 55,3 & 46,7 & 14,0 & 98.707 & 55,3 & 47,2 & 14,3 & 0,74 \\
\hline Hombres & 3.613 & 44,8 & $62,4^{2 *}$ & 16,4 & 76.044 & 44,7 & 50,2 & 15,1 & 79.657 & 44,7 & 50,8 & 15,4 & 0,79 \\
\hline Total & 8.063 & 100 & 59,6 & 16,2 & 170.301 & 100 & 48,3 & 14,6 & 178.364 & 100 & 48,8 & 14,9 & 0,76 \\
\hline
\end{tabular}

Notas. ${ }^{1} \mathrm{t}=44,2 .{ }^{2} \mathrm{t}=43,8 .{ }^{*} \mathrm{p}<0,001$. Se excluyeron del análisis 26.236 casos $(12,8 \%)$ sin datos.

Cuadro 3

Porcentaje de aciertos obtenido por los aspirantes usuarios vs no usuarios de Pruéb@te en el examen de ingreso en los concursos de selección 2018 de la UNAM, por rangos de edad

\begin{tabular}{|c|c|c|c|c|c|c|c|c|c|c|c|c|c|}
\hline & \multicolumn{4}{|c|}{ Usuarios } & \multicolumn{4}{|c|}{ No usuarios } & \multicolumn{4}{|c|}{ Total } & \multirow{2}{*}{ d } \\
\hline & $\mathrm{N}$ & $\%$ & M & $\mathrm{DE}$ & $\mathrm{N}$ & $\%$ & M & $\mathrm{DE}$ & $\mathrm{N}$ & $\%$ & M & $\mathrm{DE}$ & \\
\hline$<18$ & 1.389 & 17,2 & $58,2^{1 *}$ & 16,4 & 43.099 & 25,3 & 45,7 & 13,6 & 44.488 & 24,9 & 46,1 & 13,8 & 0,90 \\
\hline 18 & 1.816 & 22,5 & $62,3^{2 *}$ & 16,8 & 45.318 & 26,6 & 47,9 & 14,7 & 47.134 & 26,4 & 48,5 & 15,1 & 0,95 \\
\hline 19 & 1.067 & 13,2 & $64,0^{3 *}$ & 16,8 & 25.207 & 14,8 & 49,6 & 15,7 & 26.274 & 14,7 & 50,2 & 16,0 & 0,90 \\
\hline 20 & 575 & 7,1 & $62,5^{4 *}$ & 16,4 & 14.026 & 8,2 & 50,4 & 15,6 & 14.601 & 8,2 & 50,9 & 15,8 & 0,76 \\
\hline$>20$ & 3.216 & 39,9 & $56,7^{5 *}$ & 14,9 & 42.651 & 25,0 & 49,9 & 14,1 & 45.867 & 25,7 & 50,4 & 14,3 & 0,47 \\
\hline Total & 8.063 & 100 & 59,6 & 16,2 & 170.301 & 100 & 48,3 & 14,6 & 178.364 & 100 & 48,8 & 14,9 & 0,76 \\
\hline
\end{tabular}

Notas. ${ }^{1} \mathrm{t}=28,1 .{ }^{2} \mathrm{t}=36,0 .{ }^{3} \mathrm{t}=27,5 .{ }^{4} \mathrm{t}=17,4 .{ }^{5} \mathrm{t}=25,0 .{ }^{*} \mathrm{p}<0,001$. Se excluyen del análisis 26.236 casos $(12,8 \%)$ sin datos.

Comparación del rendimiento en el examen de ingreso 2018 de la UNAM de los usuarios y no usuarios, por tipo de sostenimiento y bachillerato

Por el tipo de sostenimiento y bachillerato, los usuarios obtuvieron un porcentaje medio de aciertos mayor que los no usuarios, lo cual resultó estadísticamente significativo. En los usuarios, el porcentaje medio de aciertos en el examen de ingreso favoreció a las escuelas privadas $(59,1 \%)$ versus las escuelas públicas con $57 \%$ (Cuadro 4). 
Comparación del rendimiento en el examen de ingreso 2018 de la UNAM de los usuarios y no usuarios, por área de conocimiento

Como puede observarse en el Cuadro 5, los usuarios de la Web App en cada área de conocimiento, lograron los porcentajes medios de aciertos más altos en comparación con los no usuarios, las diferencias fueron estadísticamente significativas. El área con la media del porcentaje de aciertos más alta fue Ciencias Físico-Matemáticas y de las Ingenierías (CFMI), misma que cuenta con el tamaño del efecto más grande $(\mathrm{d}=0,99)$.

En el Cuadro 6 se observa que la media del porcentaje de aciertos obtenida por los usuarios según el nivel de demanda de la licenciatura es significativamente mayor que la de los no usuarios. Poco más del $60 \%$ de los aspirantes solicitan licenciaturas de demanda Media y Baja, mientras que menos del $10 \%$ de Muy alta demanda, en esta categoría solo $4,2 \%$ de los usuarios fue aceptado en contraste con el 1,9\% de los no usuarios, la diferencia resultó estadísticamente significativa y el tamaño del efecto moderado.

Comparación de los usuarios y no usuarios aceptados en 2018 en alguna de las licenciaturas de la UNAM

De los 178.364 aspirantes en los concursos de selección de 2018, solo el 13,2\% fue aceptado en alguna de las licenciaturas de la UNAM. El porcentaje de usuarios de Pruéb@te aceptado fue del 29,5\%, mientras que, en el grupo de los no usuarios, fueron aceptados el 12,5\%. Esto es, quienes emplearon la Web App representan cerca de 17 puntos porcentuales más que aquellos que no la usaron, pero también fueron aceptados. La diferencia entre el porcentaje medio de aciertos entre usuarios y no usuarios aceptados fue estadísticamente significativa (Cuadro 7).

Cuadro 4

Porcentaje de aciertos obtenido por los aspirantes usuarios vs no usuarios de Pruéb@te en el examen de ingreso en los concursos de selección 2018 de la UNAM, por sostenimiento y tipo de bachillerato

\begin{tabular}{|c|c|c|c|c|c|c|c|c|c|c|c|c|c|}
\hline & \multicolumn{4}{|c|}{ Usuarios } & \multicolumn{4}{|c|}{ No usuarios } & \multicolumn{4}{|c|}{ Total } & \multirow{2}{*}{ d } \\
\hline & $\mathrm{N}$ & $\%$ & M & $\mathrm{DE}$ & $\mathrm{N}$ & $\%$ & $\mathrm{M}$ & $\mathrm{DE}$ & $\mathrm{N}$ & $\%$ & M & $\mathrm{DE}$ & \\
\hline Pública & 4.263 & 100,0 & $57,0^{1 *}$ & 15,2 & 110.267 & 100,0 & 46,8 & 13,6 & 114.530 & 100,0 & 47,2 & 13,8 & 0,74 \\
\hline UNAM & 986 & 23,1 & $65,7^{2 *}$ & 13,1 & 10.702 & 9,7 & 60,0 & 13,1 & 11.688 & 10,2 & 60,5 & 13,2 & 0,43 \\
\hline $\begin{array}{l}\text { Otro bachillera- } \\
\text { to público }\end{array}$ & 3.277 & 76,9 & $54,4^{3 *}$ & 14,8 & 99.565 & 90,3 & 45,4 & 12,9 & 102.842 & 89,8 & 45,7 & 13,1 & 0,69 \\
\hline Privada & 4.021 & 100,0 & $59,1^{4 *}$ & 15,8 & 63.425 & 100,0 & 49,6 & 14,7 & 67.446 & 100,0 & 50,2 & 14,9 & 0,63 \\
\hline $\begin{array}{l}\text { Incorporado } \\
\text { UNAM }\end{array}$ & 656 & 16,3 & $65,3^{5 *}$ & 15,9 & 9.189 & 14,5 & 55,3 & 15,5 & 9.845 & 14,6 & 56,0 & 15,7 & 0,63 \\
\hline $\begin{array}{l}\text { Otro bachillera- } \\
\text { to privado }\end{array}$ & 3.365 & 83,7 & $57,9^{6 *}$ & 15,5 & 54.236 & 85,5 & 48,6 & 14,3 & 57.601 & 85,4 & 49,2 & 14,5 & 0,63 \\
\hline Desconocido & 56 & 100,0 & 64,2 & 17,3 & 428 & 100,0 & 57,1 & 15,7 & 484 & 100,0 & 57,9 & 16,1 & 0,44 \\
\hline
\end{tabular}

Notas. ${ }^{1} \mathrm{t}=43,2 \cdot{ }^{2} \mathrm{t}=13,1 \cdot{ }^{3} \mathrm{t}=34,4 \cdot{ }^{4} \mathrm{t}=37,1 \cdot{ }^{5} \mathrm{t}=15,6 \cdot{ }^{6} \mathrm{t}=33,9 .{ }^{*} \mathrm{p}<0,001$. 
Cuadro 5

Porcentaje de aciertos obtenido por los aspirantes usuarios vs no usuarios de Pruéb@te en el examen de ingreso en los concursos de selección 2018 de la UNAM, según el área de conocimiento

\begin{tabular}{|c|c|c|c|c|c|c|c|c|c|c|c|c|c|}
\hline & \multicolumn{4}{|c|}{ Usuarios } & \multicolumn{4}{|c|}{ No usuarios } & \multicolumn{4}{|c|}{ Total } & \multirow{2}{*}{$\mathrm{d}$} \\
\hline & $\mathrm{N}$ & $\%$ & M & $\mathrm{DE}$ & $\mathrm{N}$ & $\%$ & M & $\mathrm{DE}$ & $\mathrm{N}$ & $\%$ & M & $\mathrm{DE}$ & \\
\hline CFMI & 1.508 & 18,7 & $65,6^{1 *}$ & 16,8 & 32.411 & 19,0 & 49,8 & 15,6 & 33.919 & 19,0 & 50,5 & 16,0 & 0,99 \\
\hline CBQS & 2.550 & 31,6 & $59,2^{2 *}$ & 16,6 & 59.492 & 34,9 & 47,0 & 14,5 & 62.042 & 34,8 & 47,5 & 14,8 & 0,82 \\
\hline CS & 2.861 & 35,5 & $56,8^{3 *}$ & 15,0 & 58.874 & 34,6 & 48,7 & 14,1 & 61.735 & 34,6 & 49,1 & 14,2 & 0,57 \\
\hline HA & 1.144 & 14,2 & $59,5^{4 *}$ & 15,4 & 19.524 & 11,5 & 48,6 & 14,4 & 20.668 & 11,6 & 49,2 & 14,7 & 0,74 \\
\hline Total & 8.063 & 100,0 & 59,6 & 16,2 & 170.301 & 100,0 & 48,3 & 14,6 & 178.364 & 100,0 & 48,8 & 14,9 & 0,76 \\
\hline
\end{tabular}

Notas. ${ }^{1} \mathrm{t}=35,8 .{ }^{2} \mathrm{t}=11,7 .{ }^{3} \mathrm{t}=28,3 .{ }^{4} \mathrm{t}=23,3 .{ }^{*} \mathrm{p}<0,001$. Se excluyeron del análisis 22.140 casos $(10,8 \%)$ sin datos. CFMI $=$

Ciencias Físico Matemáticas e Ingenierías; $\mathrm{CBQS}$ = Ciencias Biológicas, Químicas y de la Salud; CS = Ciencias Sociales; HyA

= Humanidades y Artes.

Cuadro 6

Porcentaje de aciertos obtenido por los aspirantes usuarios vs no usuarios de Pruéb@te en el examen de ingreso en los concursos de selección 2018 de la UNAM, según el nivel de demanda de la licenciatura elegida

\begin{tabular}{|c|c|c|c|c|c|c|c|c|c|c|c|c|c|c|c|c|}
\hline & \multicolumn{5}{|c|}{ Usuarios } & \multicolumn{5}{|c|}{ No usuarios } & \multicolumn{5}{|c|}{ Total } & \multirow{2}{*}{ d } \\
\hline & $\mathrm{N}$ & $\%$ & $\mathrm{~A}^{\mathrm{a}}$ & M & $\mathrm{DE}$ & $\mathrm{N}$ & $\%$ & $\mathrm{~A}^{\mathrm{a}}$ & M & $\mathrm{DE}$ & $\mathrm{N}$ & $\%$ & $\mathrm{~A}^{\mathrm{a}}$ & $\mathrm{M}$ & $\mathrm{DE}$ & \\
\hline Muy baja & 941 & 11,7 & 48,0 & $61,8^{1 *}$ & 15,1 & 13.031 & 7,7 & 29,7 & 51,8 & 14,6 & 13.972 & 7,8 & 30,9 & 52,4 & 14,8 & 0,68 \\
\hline Baja & 1.764 & 21,9 & 36,6 & $59,4^{2 *}$ & 16,3 & 38.733 & 22,7 & 15,4 & 48,5 & 14,2 & 40.497 & 22,7 & 16,3 & 49,0 & 14,5 & 0,75 \\
\hline Media & 3.260 & 40,4 & 27,2 & $58,7^{3 *}$ & 16,3 & 71.918 & 42,2 & 10,8 & 47,5 & 14,4 & 75.178 & 42,1 & 11,5 & 48,0 & 14,7 & 0,76 \\
\hline Alta & 1.480 & 18,4 & 25,2 & $58,4^{4 *}$ & 16,1 & 37.175 & 21,8 & 9,3 & 47,6 & 14,7 & 38.655 & 21,7 & 9,9 & 48,0 & 14,9 & 0,73 \\
\hline Muy alta & 618 & 7,7 & 4,2 & $64,1^{5 *}$ & 16,9 & 9.444 & 5,5 & 1,9 & 52,1 & 16,1 & 10.062 & 5,6 & 2,0 & 52,8 & 16,4 & 0,73 \\
\hline Total & 8.063 & 100,0 & 29,5 & 59.6 & 16,2 & 170.301 & 100,0 & 12,5 & 48,3 & 14,6 & 178.364 & 100,0 & 13,2 & 48,8 & 14,9 & 0,76 \\
\hline
\end{tabular}

Notas. ${ }^{1} \mathrm{t}=19,7 \cdot{ }^{2} \mathrm{t}=27,6 \cdot{ }^{3} \mathrm{t}=12,4 \cdot{ }^{4} \mathrm{t}=8,1 \cdot{ }^{5} \mathrm{t}=17,1 .{ }^{*} \mathrm{p}<0,001 . \mathrm{A}^{\mathrm{a}}=$ Porcentaje de aceptación a alguna licenciatura. Todas las diferencias resultaron estadísticamente significativas con $\mathrm{p}<0,001$.

Cuadro 7

Porcentaje de usuarios vs no usuarios de Pruéb@te que ingresaron a alguna licenciatura en los concursos de selección 2018 de la UNAM

\begin{tabular}{|c|c|c|c|c|c|c|c|c|c|c|c|c|c|}
\hline & \multicolumn{4}{|c|}{ Usuarios } & \multicolumn{4}{|c|}{ No usuarios } & \multicolumn{4}{|c|}{ Total } & \\
\hline & $\mathrm{N}$ & $\%$ & M & $\mathrm{DE}$ & $\mathrm{N}$ & $\%$ & M & $\mathrm{DE}$ & $\mathrm{N}$ & $\%$ & M & $\mathrm{DE}$ & \\
\hline Muy baja & 2.382 & 29,5 & $71,5^{1 *}$ & 13,4 & 21.216 & 12,5 & 67,9 & 13,0 & 23.598 & 13,2 & 68,2 & 13,1 & 0,28 \\
\hline Muy alta & 5.681 & 70,5 & $54,6^{2 *}$ & 14,6 & 149.085 & 87,5 & 45,5 & 12,6 & 154.766 & 86,8 & 45,9 & 12,8 & 0,71 \\
\hline Total & 8.063 & 100,0 & 59,6 & 16,2 & 170.301 & 100,0 & 48,3 & 14,6 & 178.364 & 100,0 & 48,8 & 14,9 & 0,76 \\
\hline
\end{tabular}

Notas. ${ }^{1} \mathrm{t}=12,5 .{ }^{2} \mathrm{t}=46,3 .{ }^{*} \mathrm{p}<0,001$. 


\subsection{Rendimiento de los usuarios en el examen de ingreso 2018 de la UNAMy el uso de Pruéb@te}

Como se mencionó, en Pruéb@te se cuenta con el módulo Práctica de exámenes, en el cual se observó que los usuarios que completaron hasta cinco pruebas y fueron aceptados lograron más del $75 \%$ de aciertos en el examen de admisión, a diferencia del $66 \%$ que no completó exámenes de práctica. La misma situación se dio en los usuarios no aceptados, con aproximadamente $64 \%$ y $52 \%$, respectivamente. En este sentido, el porcentaje medio de aciertos incrementa con cada examen de práctica completado. Las diferencias fueron estadísticamente significativas (Figura 2).

Figura 2

Rendimiento de los usuarios de Pruéb@te en el examen de ingreso 2018 según el número de exámenes completados en la Web App

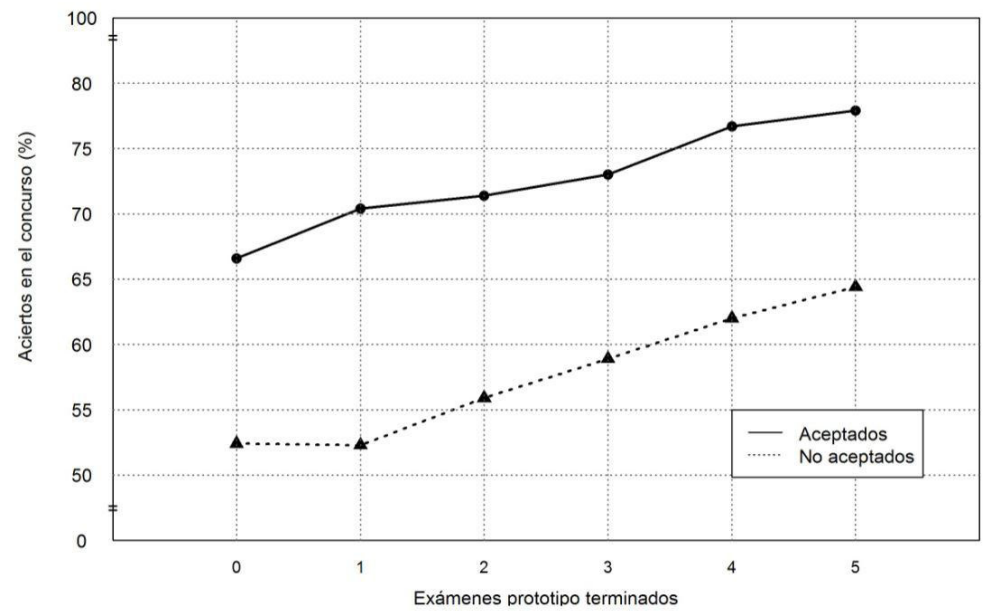

Notas. $\rho=0,29 . \mathrm{p}<0,000$

La Figura 3 muestra el porcentaje medio de aciertos obtenidos en el examen de ingreso por los usuarios aceptados y no aceptados, según el número de horas de práctica en la Web App. A medida que incrementaron las horas de práctica en Pruéb@te, alcanzaron medias en el porcentaje de aciertos más altas, de 68,4 a 74,4 y de 49,7 a 60,5 , respectivamente.

Figura 3

Rendimiento de los usuarios de Pruéb@te en el examen de ingreso 2018 según las horas de práctica

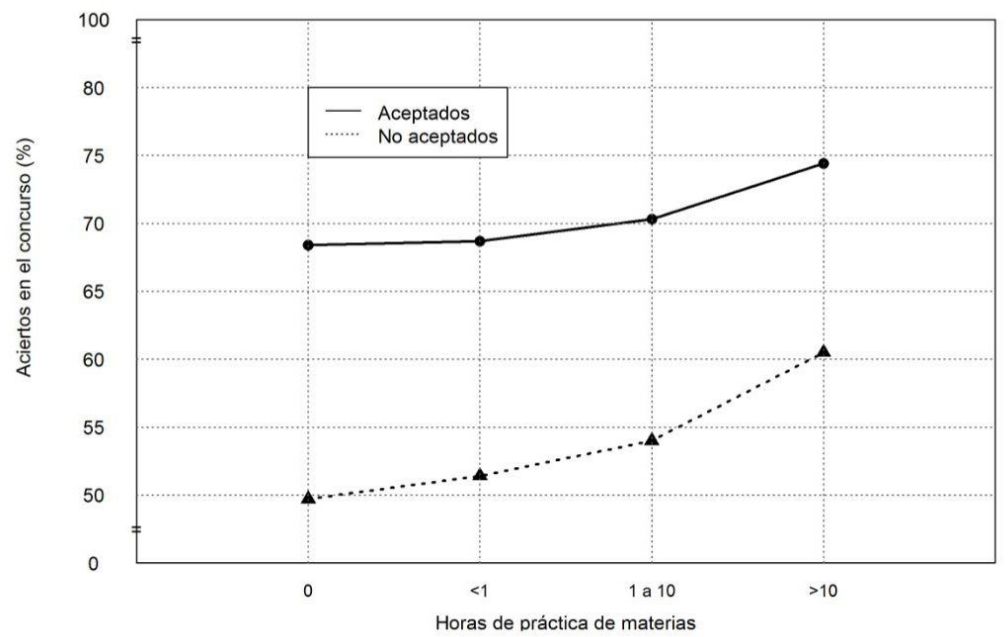

Notas. $\rho=0,29 . \mathrm{p}<0,000$. 


\subsection{Variables de mayor aporte a los resultados en el examen de ingreso a la licencia- tura}

Para determinar si el uso de Pruéb@te explica mejores resultados en el examen de ingreso a la licenciatura, se utilizó el modelo de regresión lineal múltiple. La variable dependiente fue el porcentaje de aciertos en el examen de ingreso y las variables independientes fueron: exámenes completados en Pruéb@ te; materias estudiadas; tiempo de práctica de materias; concursos de selección, sexo y edad en la última aplicación. Se realizó un modelo con todos los usuarios de Pruéb@te que fueron aceptados en alguna licenciatura, así como cinco más por cada nivel de demanda de la licenciatura elegida, en total se corrieron seis modelos. El Cuadro 8 indica los resultados del análisis. El modelo que incluyó a todos los usuarios de Pruéb@te fue significativo, $\mathrm{r}^{2}=0,173, \mathrm{p}<0,001$.

Cuadro 8

Modelo de regresión del porcentaje de aciertos en el examen de ingreso en los concursos de selección 2018 de la UNAM

\begin{tabular}{|c|c|c|c|c|c|c|}
\hline & \multirow{2}{*}{ Todos } & \multicolumn{5}{|c|}{ Nivel de demanda } \\
\hline & & Muy baja & Baja & Media & Alta & Muy alta \\
\hline$r^{2}$ & 0,173 & 0,115 & 0,170 & 0,198 & 0,172 & 0,258 \\
\hline ANOVA & $* * *$ & $* * *$ & $* * *$ & $* * \star$ & $* * *$ & $* * *$ \\
\hline \multicolumn{7}{|l|}{ Coeficientes estandarizados } \\
\hline Exámenes en Pruéb@te & $0,204^{* * *}$ & $0,165^{* * *}$ & 0,231 & $0,219^{* * *}$ & $0,179^{* * * *}$ & $0,174^{* * *}$ \\
\hline Materias estudiadas & $0,118^{* \star *}$ & 0,064 & $0,132^{* * *}$ & $0,103^{* * *}$ & $0,147^{* \star *}$ & $0,129^{* *}$ \\
\hline Tiempo de práctica & $0,112^{* * *}$ & $0,130^{* *}$ & $0,107^{* * *}$ & $0,124^{* * *}$ & $0,061^{*}$ & $0,138^{* \star *}$ \\
\hline Concursos de selección & $0,065^{* * *}$ & $-0,069^{*}$ & $-0,023$ & $0,080^{\star * \star}$ & $0,138^{* * *}$ & $0,258^{* * *}$ \\
\hline Sexo & $0,146^{* * *}$ & $0,167^{* * *}$ & $0,107^{* * *}$ & $0,167^{* * *}$ & $0,139^{* * *}$ & $0,163^{* \star *}$ \\
\hline Edad & $-0,132^{* * *}$ & $-0,084^{* *}$ & $-0,130^{* * *}$ & $-0,144^{* * *}$ & $-0,129^{* * *}$ & 0,056 \\
\hline
\end{tabular}

Notas. ${ }^{*} \mathrm{p}<0,05 ;{ }^{* *} \mathrm{p}<0,01 ;{ }^{* * *} \mathrm{p}<0,001$

Los modelos que se ejecutaron por cada nivel de demanda resultaron significativos, los valores $r^{2}$ fueron 0,115 (Muy baja), 0,170 (Baja), 0,198 (Media), 0,172 (Alta) y 0,258 (Muy alta). Los resultados del modelo que incluyó a todos los usuarios de Pruéb@te y los correspondientes a los niveles de demanda Baja, Media y Alta indicaron que la variable exámenes completados en Pruéb@te explica significativamente el aumento en los resultados en el examen de ingreso a la licenciatura, con coeficientes de correlación de 0,204, 0,231, 0,219 y 0,179, respectivamente. En el caso del modelo para el nivel de demanda Muy baja, la variable Sexo del alumno fue la mejor predictora, con un coeficiente de correlación de 0,167, p < 0,001. Mientras que, para el nivel de demanda Muy alta, fue la variable Concursos de selección con un coeficiente de $0,258, p<0,001$. La variable Edad en la última aplicación obtuvo un coeficiente de correlación negativo y significativo en los distintos modelos, excepto para el del nivel de Muy alta demanda. Esto significa que, a mayor edad, menor puntaje se obtendrá en el examen de admisión.

A partir de los resultados, se observa que la práctica a partir de exámenes de prueba se asocia con puntajes mayores en el examen de admisión. Por otro lado, quienes ingresaron a licenciaturas de muy alta demanda, la práctica, entendida como la participación en más de un curso de selección, contribuyó a un puntaje mayor en el examen y con ello la aceptación a la licenciatura elegida.

\section{Discusión y conclusiones}

Cuando el ingreso a la universidad depende de exámenes de selección, resulta importante que los estudiantes conozcan cuáles son las estrategias que se pueden utilizar para mejorar los resultados en las pruebas, así como las ganancias generales que los estudiantes obtendrán tras su preparación. En este es- 
tudio se compararon los resultados obtenidos por los aspirantes que utilizaron Pruéb@te UNAM para el examen de ingreso a la licenciatura en contraste con los que no lo utilizaron. Los hallazgos muestran que el rendimiento en el examen de selección de los aspirantes que lo utilizaron es significativamente mayor comparado con los que no lo usaron, 59,6 vs 48,3, respectivamente.

Al analizar la relación entre el uso de Pruéb@te UNAM y el puntaje del examen de admisión a la licenciatura de la UNAM con el sexo, edad, tipo de sostenimiento y bachillerato, área de conocimiento, nivel de demanda de la carrera, exámenes completados en Pruéb@te y el tiempo de uso de la plataforma (horas de práctica), los resultados apuntaron diferencias significativas entre usuarios y no usuarios en cada una de las variables, siempre a favor de los usuarios.

El porcentaje medio de aciertos en el examen de ingreso es mayor para los hombres usuarios de Pruéb@ te que para las mujeres; esto mismo aplica para los no usuarios. Lo anterior se ha encontrado en otros estudios en los que se señala que las mujeres obtienen consistentemente puntuaciones más bajas que los hombres, a pesar de que su rendimiento académico es superior y utilizan un método de preparación para la prueba (Appelrouth et al., 2017). Una explicación para esta diferencia puede atribuirse a la autoeficacia y percepción acerca de la prueba, la competencia y la ansiedad (Byrnes y Takahira 1993; Niederle y Vesterlund, 2010). En promedio, las mujeres suelen mostrar un mayor grado de ansiedad académica ante las pruebas que los hombres (Altermatt y Kim 2004; Eum y Rice 2011; Szafranski et al., 2012).

Además, como resultado del análisis del fenómeno de "discriminación de género", se han documentado las diferencias existentes en los puntajes obtenidos por hombres y mujeres en pruebas a gran escala que evalúan lenguaje y matemáticas; señalando que las diferencias son atribuibles a desigualdades dadas por las características del sistema educativo y a factores socioculturales, más que por aspectos de tipo biológico. Por lo que es importante trabajar en políticas educativas encaminadas a la equidad de género para el acceso, permanencia y regularidad en el proceso educativo (Cárcamo y Mola, 2012; Díaz et al., 2019; González et al., 2020).

Los usuarios se desempeñaron mejor en todos los rangos de edad comparados con los no usuarios. Llama la atención que a medida que incrementa la edad de los usuarios, el porcentaje medio de aciertos disminuye, esta relación también se ha identificado en variables vinculadas con el desarrollo de estrategias basadas en el aprendizaje autónomo, en este caso, los alumnos más jóvenes tienen mayor disponibilidad hacia la preparación de exámenes, trabajo intelectual sobre los contenidos y participación en el proceso de enseñanza-aprendizaje en comparación con sus pares de mayor edad (Pegalajar, 2020). En este estudio, en cinco de los seis modelos de regresión los coeficientes asociados resultaron negativos y bajos, lo que confirma, por un lado, que a mayor edad menor es el puntaje obtenido, y por otro, que la variable edad explica en menor medida el porcentaje medio de aciertos alcanzado en el examen de selección.

Con respecto al tipo de sostenimiento, se ha observado que los estudiantes que provienen de escuelas públicas logran puntajes de línea base en las pruebas más bajos y aumentos más pequeños que sus contrapartes de escuelas privadas, pero cuando se lleva a cabo el modelo lineal general en el que se consideran otros factores, la distancia entre los puntajes obtenidos por los alumnos de escuelas públicas y privadas resulta no significativa (Appelrouth et al., 2017). En este estudio los usuarios que provienen de escuelas privadas alcanzaron una media de porcentaje de aciertos mayor $(59,1 \%)$ que los de escuelas públicas (57\%), asimismo los estudiantes que provienen de bachillerato UNAM (65,7\%) e incorporadas $(65,3 \%)$, obtuvieron un mejor desempeño que los no usuarios del mismo tipo de bachillerato ( $60 \%$ y $55,3 \%$, respectivamente). No obstante, si bien se identifican diferencias en el rendimiento de los estudiantes a partir del tipo de sostenimiento de la escuela de procedencia, existe la necesidad de explorar otros factores que pueden explicar este hecho, como el estatus socioeconómico y motivación del alumno, y las relativas a los programas de preparación, entre ellas, los contenidos y la habilidad para la instrucción.

En cuanto al área de conocimiento en la que se encuentra la licenciatura que solicitó cada aspirante, la cual puede ser de Muy baja a Muy alta demanda, los resultados muestran que los aspirantes de Ciencias Físico Matemáticas e Ingenierías (CFMI) lograron el mayor porcentaje medio de aciertos (65,6\% usuarios y $49,8 \%$ no usuarios). Son los usuarios en todos los niveles de demanda quienes alcanzaron la media del porcentaje de aciertos más alta (59,6\% usuarios vs $48,3 \%$ no usuarios). Cabe señalar que menos del $6 \%$ de los aspirantes solicitan licenciaturas de muy alta demanda, de los cuales, solo $4,2 \%$ y $1,9 \%$ de los usuarios 
y no usuarios fueron aceptados, la diferencia es poco más de dos puntos porcentuales. En estas variables, Pruéb@te también se asoció con una diferencia significativa entre usuarios y no usuarios.

Bajo este panorama, utilizar un método de preparación para la presentación de exámenes se asocia con una mayor puntuación en el examen de admisión. Si bien, los examinados pueden diferir en el tipo de método utilizado y el tiempo dedicado a la preparación, los ítems de muestra o práctica y la lectura de folletos de familiarización con las pruebas se encuentran entre los más utilizados y con mejores resultados (Arendasy et al., 2016; Buchmann et al., 2010; Carrasco, 2011; Loken et al., 2004; Powers, 2012;). En el caso de los resultados de este estudio se muestra que realizar hasta cinco exámenes en Pruéb@te los cuales simulan la prueba real, se asocia con una media de porcentaje de aciertos más alta en el examen de ingreso $(77,9 \%)$ en contraste con los que no completaron alguna prueba de práctica $(66,6 \%)$, los tamaños del efecto encontrados fueron grandes y significativos, en el rango de 0,88 a 1,16. En el modelo de regresión también se observó que los exámenes completados en Pruéb@te son el factor que en mayor medida explica el porcentaje medio de aciertos en el examen de selección, sobre todo cuando la licenciatura a la que aspiran los estudiantes es de baja, media o alta demanda.

En el grupo de estudiantes que aspiran a licenciaturas de muy alta demanda, presentar el examen de ingreso en distintos concursos de selección sirvió como entrenamiento para la prueba. En el modelo de regresión que se desarrolló para este grupo, la variable concursos de selección obtuvo el coeficiente significativamente más alto al explicar el porcentaje medio de aciertos en el examen de ingreso. Estos resultados coinciden con otras investigaciones sobre el impacto de la práctica en los resultados de la prueba oficial, puede decirse que en la medida que se practica lo revisado en los programas de entrenamiento, a través de ejercicios o exámenes de simulación y en ellos se obtienen buenos resultados, es probable que la puntuación en la prueba real (examen de admisión) sea mejor en comparación con aquellos estudiantes que no entrenan (Appelrouth et al., 2017, 2018; Moore et al., 2018; Sanchez, 2019; Warne et al., 2015).

Los resultados de este estudio también revelaron que el número de horas de preparación es un factor que favorece el aumento en el porcentaje medio de aciertos de los usuarios aceptados y no aceptados, los que practicaron por más de diez horas obtuvieron $74,4 \%$ y $60,5 \%$, mientras que los que no utilizaron Pruéb@te pero sí se registraron alcanzaron 68,4\% y 49,7\%, respectivamente. Las diferencias entre los grupos fueron significativas y el tamaño del efecto para esta variable fue grande. Este hecho concuerda con incrementos en los puntajes reportados en otras investigaciones como efecto del tiempo dedicado a prepararse para el examen de admisión (Appelrouth et al., 2017; Montgomery y Lilly, 2012; Sanchez, 2019; Schaefle, 2018).

Otros efectos consecuencia de la preparación para el examen que se han reportado en la literatura y que resultaría importante explorar, tienen que ver con la posibilidad de desarrollar diversas habilidades en los estudiantes, por ejemplo, sensación de seguridad, reforzamiento y asimilación de diferentes estrategias de estudio, resistencia mental y reconocimiento de las condiciones en las que se realizan las pruebas o exámenes oficiales, lo cual les permitirá estructurar mejor su preparación en la forma más económica y eficiente para optimizar sus resultados. De esta forma, invertir en preparación de calidad redundará en beneficios al estudiante, la escuela y la comunidad (Appelrouth et al., 2018; Carrasco, 2011; Khodabakhshzadeh et al., 2017; Rosefsky et al., 2016).

Es importante mencionar las limitaciones del estudio. Los datos recopilados corresponden únicamente a los concursos de selección en 2018 de la UNAM, por lo que se requiere estudiar diferentes muestras antes de generalizar los resultados del modelo. Por otra parte, estudios de este tipo solo permiten afirmar que existe una asociación entre el desempeño en la prueba y el uso de la herramienta de preparación, mas no pretende establecer causalidad entre su uso y el ser o no seleccionado en una licenciatura. Es probable que el uso de esta aplicación sea un elemento de las estrategias que utilizan los aspirantes para su preparación. Adicionalmente, el estudio solo analizó algunas de las variables asociadas a los aspirantes como sexo y edad. Se sugiere analizar otros factores que son objeto de estudio en diferentes investigaciones, como las variables sociodemográficas de los aspirantes (estatus socioeconómico o capital cultural de los padres). No podemos descartar que la autoselección de los usuarios de la plataforma constituya un factor de motivación que influya en la diferencia entre los grupos, sin embargo, en el contexto del examen no es posible realizar una asignación aleatoria de los aspirantes a la intervención. Otra posible limitación está relacionada con actividades de preparación que los estudiantes pudieron haber realizado además del uso de Pruéb@te, las cuales pueden favorecer ganancias de puntaje obtenido en el examen. Es necesario 
recolectar información de estas variables para una mejor estimación de los efectos de la plataforma de estudio.

El estudio mostró una asociación significativa entre el uso de una herramienta digital autogestiva con un mejor rendimiento en el examen de selección de los aspirantes que la utilizaron. Dada la escasa investigación en el país sobre la preparación para los procesos de admisión, es relevante considerar estos hallazgos como tema de discusión e insumo para futuras investigaciones.

A partir de los resultados presentados es posible considerar que la preparación para el examen se encuentra dentro de un marco de referencia más amplio en el que se concibe a la evaluación como una estrategia que promueve el aprendizaje, dado que las prácticas de recuperación de información mejoran dicho proceso y muestran un efecto positivo y diferenciador en los resultados obtenidos en la prueba final, además del desarrollo de estrategias de enseñanza y de aprendizaje. Por último, es deseable realizar un seguimiento longitudinal de los estudiantes a lo largo de su trayectoria académica, para fortalecer Pruéb@te como un recurso innovador y accesible de apoyo al aprendizaje de los estudiantes.

\section{Referencias}

Altermatt, E. y Kim, M. (2004). Getting girls de-stereotyped for SAT exams. Education Digest: Essential Readings Condensed for Quick Review, 70(1), 43-48.

Appelrouth, J., Moore, D., Zabrucky, K. y Cheung, J. (2018). Preparing for high-stakes admissions tests: A moderation mediation analysis. International Research in Higher Education, 3(3), 32-50. https://doi.org/10.5430/irhe.v3n3p32

Appelrouth, J. y Zabrucky, K. (2017). Preparing for the SAT: A review. College and University, 92(1), 2-17.

Appelrouth, J., Zabrucky, K. y Moore, D. (2017). Preparing students for college admissions tests. Assessment in Education: Principles, Policy y Practice, 24(1), 78-95. https://doi.org/10.1080/0969594X.2015.1075958

Arendasy, M., Sommer, M., Gutiérrez-Lobos, K. y Fritz, J. (2016). Do individual differences in test preparation compromise the measurement fairness of admission tests? Intelligence, 55, 44-56. https://doi.org/10.1016/j.intell.2016.01.004

Brame, C. y Biel, R. (2015). Test-enhanced learning: The potential for testing to promote greater learning in undergraduate science courses. Life Sciences Education, 14(2), 1-12. https://doi.org/10.1187/cbe.14-11-0208

Briggs, D. (2009). Preparation for college admission exams. National Association for College Admission Counseling.

Buchmann, C., Condron, D. y Roscigno, V. (2010). Shadow education, American style: Test preparation, the SAT and College Enrollment. Social Forces, 89(2), 435-461. https://doi.org/10.1353/sof.2010.0105

Buzo, E., Ortega, E. y Martínez, A. (2018). Pruéb@te UNAM, opción para preparar el examen de admisión a la licenciatura. Revista Digital Universitaria, 19(6), 1-11. http://doi.org/10.22201/codeic.16076079e.2018.v19n6.a10

Byrnes, J. y Takahira, S. (1993). Explaining gender differences on SAT math items. Developmental Psychology, 29(5), 805-810. https://doi.org/10.1037/0012-1649.29.5.805

Cárcamo, C. y Mola, J. (2012). Diferencias por sexo en el desempeño académico en Colombia: Un análisis regional. Economía y Región, 6(1), 133-169.

Carrasco, S. (2011). El seminario de orientación al examen de admisión a la BUAP. Un diseño instruccional exitoso para potenciar las habilidades de razonamiento. Perfiles Educativos, 33(134), 52-64.

Díaz, K., Ravest, J. y Queupil, J. (2019). Brechas de género en los resultados de pruebas de selección universitaria en Chile. ¿Qué sucede en los extremos superior e inferior de la distribución de puntajes? Pensamiento Educativo. Revista de Investigación Educacional Latinoamericana, 56(1), 1-19. https://doi.org/10.7764/PEL.56.1.2019.5

Eum, K. y Rice, K. (2011). Test anxiety, perfectionism, goal orientation, and academic performance. Anxiety, Stress, and Coping, 24(2), 157-172. https://doi.org/10.1080/10615806.2010.488723

González, G., Nogueira, F., del Valle, M. y Grossi, C. (2020). Trayectorias educativas en el marco de la implementación del ingreso irrestricto en una universidad argentina. Revista Internacional de Educación para la Justicia Social, 9(2), 109-129._https://doi.org/10.15366/riejs2020.9.2.006 
Green, M., Moeller, J. y Spak, J. (2018). Test enhanced-learning in health professions education. A systematic review. Medical Teacher, 4O(4), 337-350. https://doi.org/10.1080/0142159X.2018.1430354

Khodabakhshzadeh, H., Zardkanloo, R. y Alipoor, I. (2017). The effect of mock tests on Iranian EFL learners' test scores. International Journal of Educationy Literacy Studies, 5(3), 47-51. https://dx.doi.org/10.7575/aiac.ijels.v.5n.3p.47

Larsen, D., Butler, A. y Roedinger, H. (2008). Test-enhanced learning in medical education. Medical Education, 42, 959-966. https://doi.org/10.1111/j.1365-2923.2008.03124.x

Leeming, F. (2002). The exam-a-day procedure improves performance in psychology classes. Teaching of Psychology, 23(3), 210-212. https://doi.org/10.1207/S15328023TOP2903_06

Loken, E., Radlinski, F., Crespi, V. H., Millet, J. y Cushing, L. (2004). Online study behavior of 100,000 students preparing for the SAT, ACT and GRE. Journal Educational Computing Research, 30(3), 255-262.

Manterola, C. y Otzen, T. (2014). Estudios observacionales: Los diseños utilizados con mayor frecuencia en investigación clínica. International Journal of Morphology, 32(2), 634-645. https://doi.org/10.4067/S0717-95022014000200042

McConnell, M., St-Onge, C. y Young, M. (2015). The benefits of testing for learning on later performance. Advances in Health Sciences Education, 20, 305-320. https://doi.org/10.1007/s10459-014-9529-1

McGaghie, W. C., Downing, S. M. y Kubilius, R. (2004). What is the impact of commercial test preparation courses on medical examination performance? Teaching and Learning in Medicine, 16(2), 202-211. https://doi.org/10.1207/s15328015tlm1602_14

Montgomery, P. y Lilly, J. (2012). Systematic reviews of the effects of preparatory courses on university entrance examinations in high school-age students. International Journal of Social Welfare, 21, 3-12. https://doi.org/10.1111/j.1468-2397.2011.00812.x

Moore, R., Sanchez, E. y San Pedro, M. O. (2018). Investigating test prep impact on score gains using quasi-experimental propensity score matching. ACT, Inc.

Niederle, M. y Vesterlund, L. (2010). Explaining the gender gap in math test scores: The role of competition. Journal of Economic Perspectives, 24(2), 129-144. https://doi.org/10.1257/jep.24.2.129

OECD. (2018). Education at a glance 2018: OECD Indicators. OECD. https://doi.org/10.1787/19991487

Pegalajar, M. (2020). Estrategias de trabajo autónomo en estudiantes universitarios noveles de educación. REICE. Revista Iberoamericana sobre Calidad, Eficacia y Cambio en Educación, 18(3), 29-45. https://doi.org/10.15366/reice2020.18.3.002

Powers, D. (1999). Coaching and the SAT I. The College Board.

Powers, D. (2012). Understanding the impact of special preparation for admissions tests. En R. B. (Eds.), Advancing human assessment. The methodological, psychological and policy contributions of ETS (pp. 553-564). Springer. https://doi.org/10.1007/978-3-319-58689-2_17

Rosefsky, A., Lavore, E. y Flores-Ivich, G. (2016). The international baccalaureate diploma programme in Mexico as preparation for higher education. A Journal of Comparative and International Education, 46(3), 344-368. https://doi.org/10.1080/03057925.2014.896188

Sanchez, E. (2019). Can using ACT online prep improve score gains? ACT Research y Policy.

Sánchez-Mendiola, M. y Delgado-Maldonado, L. (2017). Exámenes de alto impacto: Implicaciones educativas. Investigación en Educación Médica, 6(21), 52-62. https://doi.org/10.1016/j.riem.2016.12.001

Schaefle, S. (2018). The relationship between GEAR UP program involvement and Latina/o students' performance on high-stakes tests. Journal of Latinos and Education, 17(3), 201-214. https://doi.org/10.1080/15348431.2017.1310653

SEP. (2019). Principales cifras del sistema educativo nacional 2018-2019. SEP.

Szafranski, D., Barrera, T. y Norton, P. (2012). Test anxiety inventory: 30 years later. Anxiety, Stress y Coping, 25(6), 667-677. https://doi.org/10.1080/10615806.2012.663490 
Warne, R., Larsen, R., Anderson, B. y Odasso, A. (2015). The impact of participation in the advanced placement program on students' college admissions test scores. The Journal of Educational Research, 108(5), 400-416. https://doi.org/10.1080/00220671.2014.917253

Winke, P. y Lim, H. (2017). The effects of test preparation on second-language listening test perfomance. Language Assessment Quarterly, 14(4), 380-397. https://doi.org/10.1080/15434303.2017.1399396

\section{Breve CV de los/as autores/as}

\section{Melchor Sánchez-Mendiola}

Médico pediatra por la Universidad del Ejército y Fuerza Aérea, México; Maestro en Educación en Profesiones de la Salud por la Universidad de Illinois en Chicago, EUA; Doctor en Ciencias de la Educación por la UNAM. Profesor de Carrera Titular C de Tiempo Completo Definitivo, División de Estudios de Posgrado, Facultad de Medicina. Actualmente Coordinador de Universidad Abierta, Innovación Educativa y Educación a Distancia (CUAIEED) de la Universidad Nacional Autónoma de México (UNAM). Participa en proyectos de evaluación educativa y educación en profesiones de la salud. Email: melchorsm@unam.mx

ORCID ID: https://orcid.org/0000-0002-9664-3208

\section{Sandra Paola Rodríguez Castillo}

Licenciada en Psicología, egresada de la Facultad de Estudios Superiores Zaragoza, Universidad Nacional Autónoma de México. Maestra en Psicología con residencia en Evaluación Educativa por la Facultad de Psicología de la Universidad Nacional Autónoma de México. Actualmente es Jefa de área de control de calidad de exámenes de selección de bachillerato en la Subdirección de Evaluación de Bachillerato y Licenciatura, Dirección de Evaluación Educativa de la CUAIEED, UNAM. Tutora virtual, certificada por la Organización de Estados Americanos (OEA).

Email: sandra_rodriguez@cuaieed.unam.mx

ORCID ID: https://orcid.org/0000-0002-4409-1739

\section{Nancy Fabiola Pérez Herrera}

Licenciada en Psicología por la Universidad Nacional Autónoma de México. Maestra en Tecnología Educativa por la Universidad Da Vinci. Profesora de asignatura en la Maestría en Educación en Ciencias de la Salud de la Facultad de Medicina, UNAM. Imparte cursos en modalidad a distancia sobre temas de evaluación educativa a gran escala y de aula, mejora de la práctica docente y gestión institucional. Actualmente es Jefa de área de control de calidad de exámenes de selección de licenciatura de la Dirección de Evaluación Educativa de la CUAIEED, UNAM.

Email: nancy_perez@cuaieed.unam.mx

ORCID ID: https://orcid.org/0000-0002-2008-3216

\section{Manuel García-Minjares}

Actuario con estudios de Maestría en Estadística e Investigación de Operaciones por la Universidad Nacional Autónoma de México (UNAM). Profesor de Estadística, Probabilidad, Matemáticas y Operaciones en los sistemas escolarizados y a distancia de la Facultad de Contaduría y Administración de la UNAM. Actualmente es Coordinador de Análisis de Resultados de Evaluación Educativa de la Dirección de Evaluación Educativa de la CUAIEED, UNAM.

Email: manuel_garcia@cuaieed.unam.mx

ORCID ID: https://orcid.org/0000-0002-9535-5917 


\section{Adrián Martínez-González}

Médico Cirujano por la Universidad Nacional Autónoma de México. Doctor en Salud Pública y Medicina Preventiva por la Universidad Autónoma de Madrid. Profesor de Carrera Titular C Tiempo Completo Definitivo, Facultad de Medicina, UNAM. Miembro de la Academia Nacional de Medicina de México y del Sistema Nacional de Investigadores. Actualmente es Director de Evaluación Educativa de la CUAIEED, UNAM. Participa en proyectos de evaluación educativa y educación en profesiones de la salud. Email: adrianmartinez38@gmail.com

ORCID ID: https://orcid.org/0000-0002-5021-9639 